\title{
RESIDUAL RESISTIVITY DUE TO VACANCIES IN ALKALI METALS
}

\author{
P.N. GajJar, B.Y. Tilakore, J.S. Luitar and A.R. JaNi \\ Department of Pliysics, Sardar Patel University \\ Vallabh Vidyanagar 388 120, Gujarat, India \\ (Received Oclober 25, 1993; revised version March 28, 1994)
}

\begin{abstract}
We have used a psendopotential techuique to examine the contribution of monovacancy, which is one of the point defects, to the resistivity of alkali metals. Two different forms of the bare-ion local pscudopotential, to describe the electron-ion interaction in metals, have been employed in the present work. Various forms of the diclectric function have been used to incorporate the exchange and corrclation cffects among the conduction electrons. Varying effect of diclectric functions on the computed results is concluded while comparing our findings with other theoretical data.
\end{abstract}

PACS numbers: 71.25.Pi, 71.55.At, 72.15.-v

\section{Introduction}

The defects are intrinsic to real crystals and determine or modify the properties of real materials. For example, the point defects such as vacancies and interstitials modify the electrical properties because they contribute to the residual resistivity.

The main source of resistivity is the scaltering of conduction electrons by phonons, which are the encrgy quanta of lattice vibrations. The other source of resistance due to lattice imperfections are: (i) replacement of one atomic species by another atomic species, (ii) disappearance of an atom (or ion) from one site with its emergence at another site, (iii) displacement of an atom around a distorted site, (iv) stacking fault etc. The resistance due to these static imperfections (and grain boundary) is called residual resistance. At low tempcratures the residual resistance due to such imperfections can be significant while at high temperatures this contribution is very small compared to that due to the scattering of conduction electrons by phonons.

The absence of atoms or ions at lattice site (i.e. vacancy) also contribute to the residual resistivity. The absence of an atom or ion at a lattice site modifies the potential at that site and acts as a perturbation to the conduction electrons. These vacancies are among the static imperfections.

Much attention has been drawn in recent years towards the study of properties of crystals containing point defects. In the present study we have used pseudopotential method to examine the contribution of monovacancy, which is one of 
the point defects, to the resistivity of alkali metals. It is well known that lattice defects and impuritics destroy the periodicity of the lattice. In many important cases the lattice distortion due to such defect can be neglected. The motivation of the present investigation comes from the fact that such problems have not been fully explored on the basis of pseudopotential theory. We have applied here the pseudopotential technique to study vacancies because it simplifies the calculations by eliminating the core states and strong potential responsible for binding them. Within the framework of the pscudopolential formulation, for low vacancy concentration and neglecting lattice distortion, the formula for monovacancy resistivity can be derived using standard scattering theory with the missing pseudopotential associated with the vacancy as $[1,2]$

$$
\rho_{v}=\frac{3}{16 \operatorname{lin} e^{2} v_{\mathrm{F}}^{2} k_{\mathrm{F}}^{4}} \int_{0}^{2 k_{\mathrm{F}}} \int_{\Omega^{\prime}} q^{3}|v(q)|^{2} \mathrm{~d} q \mathrm{~d} \Omega^{\prime}
$$

where $n$ is the ion number density, $v(q)=v^{\text {ion }}(q) / \varepsilon(q)$ is the screened form factor of electron-ion interaction, $v_{F}$ the Fermi velocity, $q$ the momentum transfer wave vector, $\Omega^{\prime}$ the solid angle in the scattcring vector space and $\varepsilon(q)$ the diclectric function. Equation (1) reveals that the resistivily $\rho_{v}$ due to vacancies will depend scnsitively on the many-electron screcning through the form factor $v(q)$. To examine this, in the present work we have calculated $\rho_{v}$ by applying two different forms of bare-ion local pseudopotentials including various dielectric functions $[1,3-8]$.

\section{Pseudopotential form factors}

The pseudopotential theory is a theory that circumvents the necds of an accurate description of the core clectrons, i.c. the clectrons occupying the completely filled shells of atoms. In a solid these elcctrons remain very localized around the atom, whereas the remaining electrons called valence electrons determine the majority of the propertics of solids. The pscudopotential has led to widespread use of the method in solid state applications. An account of the most important early contributions has becn highlighted by Pickett [10]. The problem has still attracted the attention of the recent workers cither with a view to improve the potential or the theory underlying such properties. The basic starting point in these regards is the pscudopotential form factor which consists of ionic part and the contribution from the conduction electrons resulting in the screening of the bare-ion pseudopotential.

In the present work we have used two analytical expressions for the bare-ion local pscudopotentials $[2,9]$ to describe the electron-ion interaction in metals. The first one is a bare-ion empirical form factor in $q$-space given by (in Rydberg units)

$$
\text { Model I: } v_{\mathrm{I}}^{\text {ion }}(q)=-\frac{8 \pi Z}{\Omega_{0} q^{2}}\left[\cos q r_{c}-\frac{q^{2} r_{c}^{2}}{1+q^{2} r_{c}^{2}}\right]
$$

and another one is in direct space of the form

$$
\text { Model II: } v_{\mathrm{II}}^{\text {ion }}(r)= \begin{cases}0, & r<r_{c}, \\ -\frac{2 Z}{r}+\frac{2 Z}{r} \mathrm{e}^{-r / r_{c}}, & r>r_{c} .\end{cases}
$$

The $q$-space version of model II is

$$
v_{11}^{\text {ion }}(q)=-\frac{8 \pi Z}{\Omega_{0} q^{2}}\left[\cos q r_{c}-\frac{\exp (-1) q r_{c}}{1+q^{2} r_{c}^{2}}\left(\sin q r_{c}+q r_{c} \cos q r_{c}\right)\right] .
$$


IIere $Z$ is the valency, $\Omega_{0}$ the volume per ion, $q$ the wave vector, and $r_{c}$ the parameter of the model potential. In determining the parameter of the model potentials we adopted the procedure as discussed in our recent papers $[2,9,11,12]$ where the first zero value of the form factor (at $q=q_{0}$ ) is employed. It may be noted here that the above two forms (one in $q$-space and another in $r$-space) were constructed to confirm the physical scnse regarding their capabilities for yieiding satisfactory results of some solid state properties. In particular, this can be confirmed when the parameters of the potentials are determined in the same way. Moreover, these potentials have also produced satisfactory results $[9,12]$ about susceptibility and electrical transport properties of simple metals. IIence the present investigation will supplement a comprehensive study of metallic density on the basis of two proposed forms of the local pseudopotential. The contribution to the form factor due to conduction electrons is incorporated lhrough the use of a dielectric function $\varepsilon(q)$ either by IIartrce form or by a modificd form which includes exchange and correlation effects among the conduction clectrons [1,3-8]. This exercise has been carried out by kecping in mind the fact that different forms of a screening function give varying contributions to the form factor [11-13]. Therefore, major uncertainty in form factor could be due to the use of an improper screening function even though the barc-ion pseudopotential is stabilized by determining its parameter adequately. Finally, such an investigation helps in identifying a unique combination of a bare-ion pscudopotential and its corresponding screening function. Eventually this identificd form factor could be utilized for the unified study of any metallic system.

To evaluate the above procedure, we have calculated residual resistivity due to vacancy (monovacancy resistivity) for the alkali metals by employing aforesaid model potentials I and li. In the present work we have used different dielectric functions due to IIartree [1], Iubbard-Sham [3, 4], Taylor [5], Kleinman-Langreth [6], Vashishta-Singwi [7] and Shaw [8] to infer relative merit of screening functions in the particular metal. We note here that the motivation for the present calculation of monovacancy resistivity is evident from the success of similar investigations of other clectrical transport propertics $[11,12]$ based on pscudopotential formalism.

\section{Results, discussion and conclusions}

The value of the potential parameter $r_{c}$ determined from the $q_{0}$ value and other constants used in the computation are given in Table I. For computing monovacancy resistivity, we have performed the integral in Eq. (1) in the physically valid range $0<q \leq 2 k_{\mathrm{F}}[1,14]$. The outcome of the present investigations due to both models with different screcning functions $[1,3-8]$ arc summarized in Table II along with other available theoretical valucs. Regarding $\rho_{v}$, a piece of good quantitative information has been obtained from the work of Sharma et al. [15]. They have computed the monovacancy resistivity for the alkali metals by employing different forms of the model potential. They have considered the form factors from the model potentials of Bardeen [16], Animalu and IIcine [17], Schneider and Stoll [18], Ashcroft [19], Shaw [20], Jee and IIeine [21], Giuliano-Ruggeri [22], Bortolani and Calandra [23], Sharma and Srivastava [24] and Appapillai and Williams [25]. Sen and Sen [26], Hussain and Akinlade [27] and K'lanna [28] have also used the lo- 
cal pseudopotential to obtain $\rho_{v}$. Shyu et al. [29] have determined the residual resistance due to vacancies for $\mathrm{Li}, \mathrm{Na}$ and $\mathrm{K}$ using Shaw's [20] non-local model potential.

TABLE I

Constants and parameters used in the calculation.

\begin{tabular}{l|c|c|c|c|c|c|c|c}
\hline \hline Metal & $Z$ & $\begin{array}{c}\Omega_{0} \\
\text { (a.u.) }\end{array}$ & $\begin{array}{c}k_{\mathrm{F}} \\
\text { (a.u.) }\end{array}$ & $q_{0} / 2 k_{\mathrm{F}}$ & $\begin{array}{c}r_{\mathrm{c}} \text { (I) } \\
\text { (a.u.) }\end{array}$ & $\begin{array}{c}r_{c} \text { (II) } \\
\text { (a.u.) }\end{array}$ & $A_{1}$ & $B_{1}$ \\
\hline $\mathrm{Li}$ & 1 & 144.9 & 0.5890 & 0.67 & 1.3050 & 1.7027 & 1.00714 & 0.29857 \\
$\mathrm{Na}$ & 1 & 254.5 & 0.4882 & 0.97 & 1.0875 & 1.4190 & 1.07783 & 0.28554 \\
$\mathrm{~K}$ & 1 & 481.4 & 0.3947 & 0.94 & 1.3881 & 1.8111 & 1.16730 & 0.27051 \\
$\mathrm{Rb}$ & 1 & 587.9 & 0.3693 & 0.94 & 1.4835 & 1.9357 & 1.19750 & 0.26573 \\
$\mathrm{Cs}$ & 1 & 745.5 & 0.3412 & 1.07 & 1.4107 & 1.8405 & 1.23460 & 0.26010 \\
\hline
\end{tabular}

$r_{c}(\mathrm{I})$ - parameter of the model potential I;

$r_{c}$ (II) - parameler of the model potential II;

$A_{1}$ and $B_{1}-$ Vashishta-Singwi's constants [7].

It can be seen from Table II that the results obtained from both model potentials are consistent, satisfactory and lying within the range of other theoretical findings [15, 26-29]. It may be inferred that the use of different screening functions $[1,3-8]$ affects largely the values of vacancy resistivity. Even the results obtained with IIartree dielectric function (without exchange and correlation) differ much more from those obtained with various exchange and correlation functions. In the present investigation we have found that the use of Hartree screening function [1] gives the lowest values of monovacancy resistivity while Shaw's screening function [8] has yielded relatively higher values. Shyu et al. [29] have also obtained varying effect of exchange and correlation on the monovacancy resistivity of $\mathrm{Li}$, $\mathrm{Na}$ and K. From the response of the dielectric functions it follows that it is absolutely necessary to examine the stability of the form factor before its application for the study of such properties. IIence the nature of the screening in a particular metal could be established.

Further, it is intercsting to point out here that in spite of relatively simple application of the pseudopotcntial technique in the study of point defects, there have been only a few attempts $[2,15,26-29]$ to calculate the contribution of point defects to the electrical resistivity of different metals using the same pseudopotential. Therefore, testing of various pseudopotentials proposed so far with the same approximation in the calculation of monovacancy resistivity might give additional information.

Unfortunately, detailed experimental data are not a vailable for alkali metals about monovacancy resistivity to work out a quantitative comparison. Therefore, we have compared our values with other available theoretical data in Table II. Finally, we conclude that the calculation of monovacancy resistivity based on the pseudopotential formalism can also be regarded as one of the sensitive tests for the proper assessment of the form factor and in the absence of experimental 
TABLE II

Monovacancy resistivity of alkali metals (in $\mu \Omega \mathrm{cm} / \mathrm{at} \%$ ).

\begin{tabular}{|c|c|c|c|c|c|c|}
\hline & & $\mathrm{Li}$ & $\mathrm{Na}$ & $\mathbf{K}$ & $\mathbf{R b}$ & Cs \\
\hline \multicolumn{7}{|c|}{ Present results with model-I and different dielectric functions } \\
\hline $\mathrm{H}$ & {$[1]$} & 0.54763 & 0.77680 & 0.98855 & 1.09495 & 1.88841 \\
\hline II-S & {$[3,1]$} & 0.65335 & 0.95649 & 1.21361 & 1.34320 & 2.34519 \\
\hline $\mathrm{T}$ & [5] & 0.89935 & 1.22997 & 1.65443 & 1.87720 & 3.65895 \\
\hline $\mathbf{K}-\mathbf{L}$ & [6] & 0.73690 & 1.02326 & 1.28733 & 1.42222 & 2.51776 \\
\hline $\mathrm{V}-\mathrm{S}$ & [7] & 0.77950 & 1.17115 & 1.57465 & 1.78189 & 3.32812 \\
\hline $\mathbf{S}$ & {$[8]$} & 0.91296 & 1.49455 & 2.02588 & 2.29737 & 4.28444 \\
\hline \multicolumn{7}{|c|}{ Present results with model-II and different diclectric functions } \\
\hline H & [1] & 0.30265 & 0.60214 & 0.75953 & 0.83960 & 1.45313 \\
\hline II-S & {$[3,4]$} & 0.35946 & 0.73738 & 0.92654 & 1.02327 & 1.79455 \\
\hline $\mathrm{T}$ & [5] & 0.17532 & 0.93357 & 1.23943 & 1.40139 & 2.73374 \\
\hline K-L & {$[6]$} & 0.39861 & 0.78495 & 0.97836 & 1.07864 & 1.91696 \\
\hline $\mathrm{V}-\mathrm{S}$ & [7] & 0.42180 & 0.89451 & 1.18773 & 1.33980 & 2.51216 \\
\hline $\mathbf{S}$ & [8] & 0.49595 & 1.13683 & 1.52103 & 1.71952 & 3.23036 \\
\hline \multicolumn{7}{|l|}{ Other results } \\
\hline Bardeen & {$[16]^{*}$} & 0.19686 & 0.406260 & 0.805527 & 0.91404 & 1.15344 \\
\hline Animalu-Heine & {$[17]^{*}$} & 2.72457 & 0.207050 & 0.534177 & 0.73259 & 0.288477 \\
\hline Schneider-Stoll & {$[18]^{*}$} & 0.59212 & 0.482355 & 0.3000 & 0.414522 & 0.383076 \\
\hline Ashcroft & {$[19]^{*}$} & 0.99589 & 0.203742 & 0.45159 & 0.385776 & 0.71125 \\
\hline Shaw & {$[20]^{*}$} & 1.50489 & 0.25127 & 0.878265 & - & - \\
\hline Lee-IIcine & {$[21]^{*}$} & 0.96471 & 0.491256 & 0.171882 & 0.893079 & 1.95579 \\
\hline Giuliano-Ruggeri & {$[22]^{*}$} & 1.20051 & 0.79798 & 0.397845 & 3.6261 & 5.23780 \\
\hline \multicolumn{7}{|l|}{ Bortolani- } \\
\hline Calandra & {$[23]^{*}$} & - & - & 0.32339 & 1.20087 & 1.51452 \\
\hline Sharm-Srivastava & {$[24]^{*}$} & 0.88732 & 0.212262 & 0.562328 & 1.23525 & 1.14066 \\
\hline \multicolumn{7}{|l|}{ Appapillai- } \\
\hline Williams & {$[25]^{*}$} & 3.50629 & 0.16656 & 0.47379 & 1.88931 & 1.93097 \\
\hline Sen-Sen & {$[26]$} & - & 1.32 & 1.66 & 1.84 & - \\
\hline IIussain-Akinlade & [27] & 0.40 & 0.57 & 0.85 & 0.95 & 0.63 \\
\hline \multirow[t]{2}{*}{ Khanna } & [28] & - & 0.67 & 1.00 & 1.06 & 1.21 \\
\hline & & - & 0.77 & 0.98 & - & - \\
\hline \multirow[t]{3}{*}{ Shyu et al. } & [29] & 0.578 & 0.774 & 1.028 & - & - \\
\hline & & 0.719 & 1.061 & 1.451 & - & - \\
\hline & & 0.763 & 1.138 & 1.531 & - & - \\
\hline
\end{tabular}

* From Ref. [15]. 
information such calculations may be considered as one of the guidelines for further investigations either theoretical or experimental.

\section{R.cferences}

[1] W. IIarrison, Pseudopolcntial in the Theory of Melals, W.A. Benjamin Pub. Co., New York 1966.

[2] R.S. Avte, P.N. Gajjar, A.R. Jaui, Phys. Status Solidi B 17G, K59 (1993).

[3] J. II ubbard, Proc. R. Soc. Lond. A 243, 336 (1958).

[4] L.J. Sham, Proc. R. Soc. Lond. A 283, 33 (1965).

[5] R. Taylor, J. Phys. F, Melal Plyys. 8, 1699 (1978).

[6] L. K'leinman, D.C. Langreth, Phys. Rev. 160, 589 (1967); 172, 383 (1969).

[7] P. Vashishtıa, K.S. Singwi, Phys. Rev. B 6, 875 (1972).

[8] R.W. Slaw, J. Phys. C 2, 2335 (1969).

[9] A.R. Jani, P.N. Gajjar, II.K. Patcl, Phys. Status Solidi B 169, K105 (1992).

[10] W.E. Pickott, Comp. Phys. Rep. 0, 117 (1989).

[11] A.R. Jani, II.K. Patel, P.N. Gajjar, Indian J. Pure Appl. Phys. 31, 439 (1993).

[12] A.R. Jani, II.K. Patcl, P.N. Gajjar, G. Sajcevkumar, in: Proc. Internat. Conf. INDIAS-91, Ed. Srivastava, Indias Pub., Allahiabad(India) 1993, p. 15.

[13] A.J. Greenficld, N. Wiscr, J. Phys. F, Melal Phys. 5, 1289 (1975).

[14] J.M. Ziman, Philos. Mag. G, 1013 (1961).

[15] P.K. Sharma, Jai Prakash, K.N. Mchrotra, P.C. Sharma, Acla Phys. Pol. A 56, 765 (1979).

[16] J. Bardeen, Phys. Rev. 52, 688 (1937).

[17] A.O.E. Animalu, V. IIcinc, Philos. Mag. 12, 1249 (1965).

[18] T. Schneider, E. Stoll, Phys. Kondens. Mater 5, 331, 364 (1966); 8, 58 (1968).

[19] N.W. Ashcroft, Phys. Lell. 23, 48 (1966); J. Phys. C 1, 232 (1968).

[20] R.W. Shaw, Phys. Rev. 174, 769 (1968).

[21] M.J.G. Lee, V. IIcine, private communication; M.J.G. Lee, Phys. Rev. 178, 953 (1969).

[22] E.S. Giuliano, R. Ruggeri, Nuovo Cimenlo B 61, 53 (1969); Lelt. Nuovo Cimenlo 2, 817 (1969).

[23] V. Bortolani, C. Calandra, Phys. Rev. B 1, 2405 (1970).

[24] P.K. Sharma, S.K. Srivastava, J. Phys. Chem. Solids 33, 1979 (1972).

[25] M. Appapillai, A.R. Williams, J. Phys. F 3, 759 (1973).

[26] D. Sen, S.K. Sen, Phys. Stalus Solidi B 127, 337 (1985).

[27] L.A. Ilussain, O. Akinliade, Phys. Slatus Solidi B 130, K123 (1987).

[28] K.N. Khanna, Indian J. Phys. A 52, 552 (1978).

[29] W.M. Shyu, J.II. Welling, M.R. Cordes, G.D. Gaspari, Phys. Rev. B 4, 1802 (1971). 